\title{
THE WEAK* KARLOVITZ LEMMA FOR DUAL LATTICES
}

\section{BRaIley SIMS}

We establish the Karlovitz lemma for a nonexpansive self mapping of a nonempty weak* compact convex set in a weak* orthogonal dual Banach lattice.

We say a Banach space has the weak fixed point property (w-fpp) if every nonexpansive self mapping of a nonempty weak compact convex subset has a fixed point. In the case of a dual space we say it has the $w^{*}$-fpp if every nonexpansive self mapping of a nonempty weak*compact convex subset has a fixed point.

Let $C$ be a nonempty weak (weak*) compact convex set and let $T: C \longrightarrow C$ be a nonexpansive mapping. The weak (weak*) compactness and Zorn's lemma ensure the existence of minimal nonempty weak (weak*) compact convex subsets of $C$ which are invariant under $T$. For brevity we will refer to such a set as a weak (weak*) compact minimal invariant set for $T$. It is readily verified that a space (dual space) has the $\mathrm{w}_{\text {-fpp }}\left(\mathrm{w}^{*}\right.$-fpp) if and only if every such weak (weak*) compact minimal invariant set has precisely one element.

Fundamental for establishing the w-fpp for certain spaces has been the result of Brodskii and Mil'man [2], Garkarvi [3] and Kirk [7] that any such weak (weak*) compact minimal invariant set $D$ is diametral in the sense that, for all $x \in D$

$$
\sup _{y \in D}\|x-y\|=\operatorname{diam} D:=\sup _{x_{1}, x_{2} \in D}\left\|x_{1}-x_{2}\right\|
$$

Another useful observation has been the existence in any nonempty closed convex subset of $C$ which is invariant under $T$ of an approximate fixed point sequence for $T$, that is a sequence $\left(a_{n}\right) \subset C$ for which

$$
\left\|a_{n}-T a_{n}\right\| \longrightarrow 0
$$

(Such a sequence may be constructed by choosing $x_{0}$ in the set and taking $a_{n}$ to be the unique fixed point of the strict contraction $V_{n} x:=(1-1 / n) T x+(1 / n) x_{0}$, whose existence is ensured by the Banach contraction mapping theorem.)

Received 4th March 1991

Copyright Clearance Centre, Inc. Serial-fee code: 0004-9729/92 \$A2.00+0.00. 
In the weak case deeper more recent results (for example, Maurey [9]; Borwein and Sims [1]; Lin [8]) have relied on the Karlovitz' lemma:

(1) If $C$ is a nonempty weak compact convex set, $D \subseteq C$ is a minimal invariant set for the nonexpansive map $T: C \rightarrow C$, and $\left(a_{n}\right)$ is an approximate fixed point sequence for $T$ in $D$, then

$$
\lim _{n}\left\|x-a_{n}\right\|=\operatorname{diam} D, \quad \text { for all } x \in D \text {. }
$$

Proofs of this result (Karlovitz [5] and Goebel [4]) have involved an appeal to Mazur's theorem; that the weak and norm closures of a convex set coincide, and so left open the question of whether a similar result holds in the weak* case. This impediment to progress in the weak* case was attacked by Khamsi [6], who established a weak* Karlovitz lemma for stable duals and dual spaces with a shrinking strongly monotone Schauder basis.

The purpose of this note is to extend these results to a weak* Karlovitz lemma for weak* orthogonal dual Banach lattices.

By analogy with Borwein and Sims [1] we say that a dual lattice $X$ is weak* orthogonal if whenever $\left(x_{n}\right)$ converges weak ${ }^{*}$ to 0 we have

$$
\lim _{n}\left\|\left|x_{n}\right| \wedge|x|\right\|=0, \text { for all } x \in X .
$$

In general it may be convenient to interpret $\left(x_{n}\right)$ as a net. However in smoothable dual spaces, in particular separable dual spaces, sequences suffice.

Proofs of the Brodskii-Mil'man result and the Karlovitz lemma have directly, or indirectly, relied on an idea captured in the following lemma which was first made explicit in the weak case by Maurey [9] while proving the $w$-fpp for $c_{0}$ and reflexive subspaces of $\mathcal{L}_{1}[0,1]$.

LEMMA 1. Let $T$ be a nonexpansive mapping of a nonempty weak (weak*) compact convex set and let $D$ denote a minimal invariant set for $T$. If $\psi: D \longrightarrow \mathbf{R}$ is a weak (weak*) lower semi-continuous convex mapping with $\psi(T x) \leqslant \psi(x)$ for all $x \in D$, then $\psi$ is constant on $D$.

Proof: Since $D$ is weak (weak*) compact and $\psi$ is weak (weak*) lower semicontininuous, $\psi$ achieves its minimum on $D$. Let $x_{0} \in D$ be such that $\psi\left(x_{0}\right)=$ $\min \psi(D)$ and let $E=\left\{x \in D: \psi(x)=\psi\left(x_{0}\right)\right\}$; then $E$ is a nonempty weak (weak*) closed convex set which is invariant under $T$. Thus, by minimality $E=D$, establishing the lemma.

To illustrate how the lemma may be used we prove the result of Brodskii-Mil'man in the weak* case. A substantially simplified version of the same argument establishes the corresponding result for weak compact sets. 
THEOREM 2. If $D$ is a weak* compact minimal invariant set for a nonexpansive mapping $T$ then $D$ is diametral.

Proof: It suffices to verify that $\psi$ defined by

$$
\psi(x):=\sup \{\|x-y\|: y \in D\}
$$

satisfies the hypotheses for Lemma 1 , as then $\psi$ is a constant on $D$ with value equal to

$$
\sup _{x \in D} \psi(x)=\sup _{x \in D} \sup _{y \in D}\|x-y\|=\operatorname{diam}(D)
$$

To complete the proof we first note that, since $\|\cdot\|$ is a dual norm, $\psi$ is the supremum of weak ${ }^{*}$ lower semi-continuous functions and so is itself weak* lower semi-continuous. Next, observe that

$$
\psi(x)=\sup _{y \in \operatorname{co} T(D)}\|x-y\| .
$$

This follows, since by the minimality of $D$, we have $D=\overline{\mathrm{co}}^{\boldsymbol{w}^{*}} T(D)$, so given $\varepsilon>0$ there exists a $y_{e} \in D$ with $\psi(x)-\varepsilon \leqslant\left\|x-y_{e}\right\|$ and a net $y_{\alpha} \rightarrow^{w^{*}} y_{e}$ with $y_{\alpha} \in \operatorname{co} T(D)$. Thus,

$$
\psi(x)-\varepsilon \leqslant\left\|x-y_{\varepsilon}\right\| \leqslant \liminf _{\alpha}\left\|x-y_{\alpha}\right\|
$$

and so there exists a $y \in \operatorname{co} T(D)$ with $\psi(x)-2 \varepsilon \leqslant\|x-y\|$ establishing the claim.

It now follows by standard convexity arguments that

$$
\psi(x)=\sup _{y \in T(D)}\|x-y\|
$$

from which it is readily seen that $\psi(T x) \leqslant \psi(x)$, completing the proof.

The Karlovitz' lemma for a weak compact minimal invariant set $D$ follows from the weak lower semi-continuity of the function $\psi(x):=\lim \sup \left\|x-a_{n}\right\|$, where $\left(a_{n}\right)$ is an approximate fixed point sequence for $T$ in $D$, which in turn follows since the epigraph of $\psi$ is a norm closed convex set and hence also weak closed by Mazur's theorem.

As the following result shows, Karlovitz' lemma also holds for a weak* compact minimal invariant set $D$ whenever functions of the above form are weak* lower semicontinuous.

LEMMA 3. Let $\left(a_{n}\right)$ be an approximate fixed point sequence for the nonexpansive mapping $T$ in the weak* compact minimal invariant set $D$. If for each subsequence $\left(y_{k}\right)$ of $\left(a_{n}\right)$ the function

$$
\psi(x):=\underset{k}{\limsup }\left\|x-y_{k}\right\|
$$


is weak* lower semi-continuous on $D$, then

$$
\lim _{n}\left\|x-a_{n}\right\|=\operatorname{diam}(D), \quad \text { for all } x \in D \text {. }
$$

Proof: Let $\left(y_{k}\right)$ be any subsequence of the approximate fixed point sequence $\left(a_{n}\right)$ then lemma 1 applies to show that $\psi(x):=\limsup \left\|x-y_{k}\right\|$ is constant on $D$ with value $c$ say. Now let $\left(y_{k_{\alpha}}\right)$ be a subnet with $y_{k_{\alpha}}{ }^{k}{ }^{w *} y_{0}$; then

and so

$$
c \geqslant \underset{\alpha}{\limsup }\left\|x-y_{k_{\alpha}}\right\| \geqslant \underset{\alpha}{\liminf }\left\|x-y_{k_{\alpha}}\right\| \geqslant\left\|x-y_{o}\right\|
$$

$$
c \geqslant \sup _{x \in D}\left\|x-y_{0}\right\|=\operatorname{diam}(D), \quad \text { by Theorem } 2 \text {. }
$$

Thus for each subsequence $\left(y_{k}\right)$ of $\left(a_{n}\right)$ we have

$$
\underset{k}{\limsup }\left\|x-y_{k}\right\|=\operatorname{diam}(D)
$$

for all $x$ in $D$ and the result follows.

Unfortunately in a dual space not all functions of the form $\psi(x):=\lim \sup \left\|x-y_{n}\right\|$, even when $\left(y_{n}\right)$ is a norm one weak* null sequence, need be weak* lower semicontinuous.

EXAMPLE 4. In $\ell_{\infty}$ define $\psi$ by

where

$$
\psi(x):=\underset{n}{\lim \sup }\left\|x-y_{n}\right\|
$$

$$
y_{n}(i)= \begin{cases}0, & i=1,2, \cdots, n-1, \\ -1, & i=n, \cdots\end{cases}
$$

Then for

$$
x_{n}(i):= \begin{cases}1, & i=1, \cdots, n \\ 0, & \text { otherwise }\end{cases}
$$

we have $x_{n} \rightarrow^{w^{*}} x_{\infty}:=(1,1, \cdots, 1, \cdots)$, while $\psi\left(x_{n}\right)=1 \not \psi \psi\left(x_{\infty}\right)=2$, so $\psi$ is not weak* lower semi-continuous.

The next example, due to Simon Fitzpatrick (private communication), shows that even in separable dual spaces such a $\psi$ may not be weak* lower semi-continuous.

EXAMPLE 5. Equivalently renorm $c_{0}$ by

$$
\|(x(i))\|=\sup \{|x(1)-x(i)+x(j)|: 1 \leqslant i \leqslant j\}
$$

and let $X$ be its dual space $\left(\ell_{\infty},\|\cdot\|^{*}\right)$. 
The natural basis vectors, $e_{n}:=\left(\delta_{n i}\right)_{i=1}^{\infty}, n=1,2, \ldots$, form a norm one weak* null sequence in $X$ and we define $\psi$ by

$$
\psi(x):=\underset{n}{\lim \sup }\left\|x-e_{n}\right\|^{*}
$$

Then taking $x_{n}:=e_{n}-e_{1}$ we have $x_{n} \rightarrow^{w^{*}}-e_{1}$, while

$$
\begin{aligned}
\psi\left(x_{j}\right) & =\underset{n}{\limsup }\left\|e_{j}-e_{1}+e_{n}\right\|^{*} \\
& =1 \\
& \nrightarrow \psi\left(-e_{1}\right)=\underset{n}{\limsup }\left\|e_{1}+e_{n}\right\|^{*}=2 .
\end{aligned}
$$

Thus $\psi$ is not weak* lower semi-continuous.

On the other hand, we now show that in a weak* orthogonal dual lattice such a function $\psi$ is always weak* lower semi-continuous.

Lemma 6. Let $X$ be a weak* orthogonal dual Banach lattice and let $y_{n} \rightarrow w^{*} 0$ with $\left\|y_{n}\right\| \leqslant 1$. Then

$$
\psi(x):=\underset{n}{\limsup }\left\|x-y_{n}\right\|
$$

is weak* lower semi-continuous.

Proof: It suffices to show that for each $\lambda$ the sub-level set

$$
D_{\lambda}:=\{x: \psi(x) \leqslant \lambda\}
$$

is weak* closed. Thus, suppose $\left(x_{\alpha}\right) \subseteq D_{\lambda}$ with $x_{\alpha} \rightarrow^{w^{*}} x$, we must show that $x \in C_{\lambda}$. Now given $\varepsilon>0$ we may by the weak* orthogonality choose $\alpha_{0}$ 'sufficiently large' so that $\left\||x| \wedge\left|x_{\alpha_{0}}-x\right|\right\|<\varepsilon / 3$. Then, for all sufficiently large $n$ we have $\left\|x_{\alpha_{0}}-y_{n}\right\| \leqslant \psi\left(x_{\alpha_{0}}\right)+\varepsilon / 3$ and $\left\|\left|y_{n}\right| \wedge\left|x_{\alpha_{0}}-x\right|\right\| \leqslant \varepsilon / 3$, and so, since

we have

$$
\begin{aligned}
\left|x-y_{n}\right| & \leqslant\left|\left(x-y_{n}\right)+\left(x_{\alpha_{0}}-x\right)\right|+\left|x-y_{n}\right| \wedge\left|x_{\alpha_{0}}-x\right| \\
& =\left|x_{\alpha_{0}}-y_{n}\right|+\left|x-y_{n}\right| \wedge\left|x_{\alpha_{0}}-x\right| \\
& \leqslant\left|x_{\alpha_{0}}-y_{n}\right|+|x| \wedge\left|x_{\alpha_{0}}-x\right|+\left|y_{n}\right| \wedge\left|x_{\alpha_{0}}-x\right|
\end{aligned}
$$

$$
\left\|x-y_{n}\right\| \leqslant\left(\psi\left(x_{\alpha_{0}}\right)+\varepsilon / 3\right)+\varepsilon / 3+\varepsilon / 3
$$

$$
\leqslant \lambda+\varepsilon \text {. }
$$

It follows that $\psi(x)=\lim \sup \left\|x-y_{n}\right\| \leqslant \lambda$, as required.

We now obtain our main result as a corollary to Lemma 6 and Lemma 3, where by a suitable dilation and translation we may assume without loss of generality that $\left(a_{n}\right)$ is weak* null with $\left\|a_{n}\right\| \leqslant 1$. 
Theorem 7. Let $X$ be a weak* orthogonal dual Banach lattice and let $\left(a_{n}\right)$ be an approximate fixed point sequence for the nonexpansive mapping $T$ in the weak* compact minimal invariant set $D$, then

$$
\lim _{n}\left\|x-a_{n}\right\|=\operatorname{diam}(D), \text { for all } x \in D
$$

Since the condition of Opial is a geometric analogue of weak orthogonality, Sims [10], we are led to ask: is a weak* Karlovitz' lemma true for dual spaces satisfying the weak* Opial condition?

We conclude by observing that this result combined with analogous arguments in the weak* case to those in Sims [10] establish the weak*-fpp for weak* orthogonal dual lattices, a result which in part subsumes the conclusions of Soardi [11], and Khamsi [6].

\section{REFERENCES}

[1] J. Borwein and B. Sims, 'Non-expansive mappings on Banach lattices and related topics', Houston J. Math. 10 (1984), 339-355.

[2] M.S. Brodskii and D.P. Mil'man, 'On the center of a convex set', Dokl. Akad. Nauk. SSSR 59 (1948), 837-840.

[3] A.L. Garkavi, 'The best possible net and the best possible cross-section of a set in a normed linear space', Amer. Math. Soc. Trans. Ser. 239 (1964), 111-131. .

[4] K. Goebel, 'On the structure of minimal invariant sets for nonexpansive mapings', Ann. Univ. Mariae Curie-Sktodowska Sect A (Lublin) 9 (1975), 73-77.

[5] L.A. Karlovitz, 'Existence of fixed points of nonexpansive mappings in a space without normal structure', Pacific J. Math. 66 (1976), 153-159.

[6] M.A. Khamsi, 'On the weak*-fixed point property', Contemp. Math. 85 (1989), 325-337.

[7] W.A. Kirk, 'A fixed point theorem for mappings which do not increase distances', Amer. Math. Monthly 72 (1965), 1004-1006.

[8] P-K. Lin, 'Unconditional bases and fixed points of nonexpansive mappings', Pacific $J$. Math. 116 (1985), 69-76.

[9] B. Maurey, 'Seminaire d'Analyse Fonctionnelle', Exposé No. VIII (1980).

[10] B. Sims, 'Orthogonality and fixed points of nonexpansive maps', Proc. Centre Math. Anal. Aust. Nat. Uni. 20 (1988), 178-186.

[11] P. Soardi, 'Existence of fixed points of nonexpansive mappings in certain Banach lattices', Proc. Amer. Math. Soc. 73 (1979), 25-29.

Deaprtment of Mathematics The University of Newcastle

New South Wales 2308

Australia 